\title{
Field measurements of WSD-DTT protection ratios over outdoor and indoor reference geometries
}

\author{
Pekka Talmola $^{1}$, Juha Kalliovaara ${ }^{2}$, Jarkko Paavola $^{3}$, Reijo Ekman $^{3}$, Atte Vainisto ${ }^{3}$, Niko Aurala ${ }^{3}$, \\ Heikki Kokkinen ${ }^{4}$, Kari Heiska ${ }^{5}$ Risto Wichman ${ }^{6}$, Jussi Poikonen ${ }^{6}$ \\ ${ }^{1}$ Nokia, Finland, ${ }^{2}$ University of Turku, Finland, ${ }^{3}$ Turku University of Applied sciences, Finland, \\ ${ }^{4}$ Fairspectrum, Finland, ${ }^{5}$ Digita, Finland, ${ }^{6}$ Aalto University, Finland \\ Contact: jussi.poikonen@aalto.fi
}

\begin{abstract}
A currently prevalent view of the operation of white space cognitive radios is that they should use geolocation databases to determine their frequency band and maximum transmission power in any given location so that interference radiated to broadcast receivers is negligible. To estimate the level of such interference, physical reference geometries and path loss estimates have been proposed between co-located devices. In this paper we present results of a measurement campaign conducted over indoor and outdoor reference geometry scenarios in a digital terrestrial television test network. Applying a subjective error criterion, we provide practical estimates on the maximum relative transmit powers for white space devices in order to avoid visible interference in television reception. The study applies to various primary and secondary transmission and reception scenarios, and its results can be extended also to estimate protection ratios for signal configurations not considered in the reported measurements.
\end{abstract}

\section{INTRODUCTION}

The demands set for wireless connectivity are ever increasing both in terms of available data rates and geographical coverage. On the other hand, wireless communication technologies are gradually approaching the fundamental theoretical limits of the bandwidth efficiency. Thus to satisfy the increasing connectivity demands, it is in practice necessary to utilize existing frequency resources more efficiently. Currently for any given geographical location there is unused spectrum within for example the range of frequencies reserved for television broadcasts. The term cognitive radio refers to adaptive radio systems which can adjust their operating parameters according to the radio environment. Cognitive radios could conceivably utilize white spaces - frequencies allocated to communication services such as broadcasting, but which are not used locally. We refer to such cognitive radios as white space devices (WSD).

However, flexible spectrum allocation regulations are needed to enable the usage of cognitive radio. The existing licensed users in the radio spectrum are the primary users whose services need to be protected from interference from the secondary cognitive users. The interference caused by cognitive radios needs to be studied to acquire realistic protection criteria for the primary users. In [1] protection ratio (PR) is defined as the minimum value of the signal-to-interference ratio required to obtain a specific reception at the receiver input. In this paper the reception quality roughly corresponds to the ESR5 criterion [2] and the protection ratio is expressed as the ratio of the useful signal power $\left(P_{p r i}\right)$ to the interfering power from a cognitive radio system $\left(P_{w s d}\right)$ received by a primary user, $\frac{P_{p r i}}{P_{w s d}}$.

Various methodologies have been chosen to develop protection criteria for each of the incumbent services studied. Some of these protection criteria have been developed using results of measurements whilst others have been developed using parameters from ECC, ITU or ETSI deliverables. This has resulted in a number of different recommendations for the technical and operational requirements that should be applied to WSDs operating in the frequency band $470-790 \mathrm{MHz}$ in order to protect the incumbents that are TV transmissions and Programme Making and Special Events (PMSE) equipment.

Technical work to define European-wide protection ratio proposals have been performed in CEPT ECC group SE43 [3]. Three different methods for the identification of the white space exist: sensing, geolocation database and beacon signal [4]. Each of the methods has its drawbacks, but the combination of geolocation and sensing techniques might be the most accurate option. However, frequency regulators in the USA [5] and in the UK [6] are confident that geolocation database can provide sufficient protection for incumbents. In a geolocation database model the WSD would be provided with the information about the available frequencies and the maximum power levels that could be used on the frequencies. In practice, the geolocation database may contain coverage maps of the primary Digital Terrestrial Television (DTT) networks; these maps have some limited spatial resolution or pixel size - and the database must calculate the allowed transmission power for a WSD located within a given pixel of the coverage map. The maximum power levels in the database could be updated with the information obtained from the spectrum sensing performed by the WSD [7].

Two cases must be distinguished when considering the interference caused by a WSD to DTT reception. In the first case the WSD and the victim DTT receiver are separated by a relatively large distance and interference is primarily of cochannel type. The DTT receiver location can be estimated as the nearest possible pixel of the DTT service area in question, and the coupling loss between the WSD and DTT receiver can be calculated based on their distance and a suitable 
propagation model.

In the second case the WSD and the victim DTT receiver are within the same pixel. In this case the interference is typically of adjacent channel type, as co-channel operation is not possible within the DTT service area. Calculating the coupling loss in this case is problematic as nothing is known about the geometry between the WSD and the DTT receiver, only that they may exist within the same pixel and thus their distance is constrained by the pixel dimensions. This problem is discussed in the ECC Report 159 [4], and a proposed solution is to use a reference geometry in the calculation of coupling loss between the devices. Several different reference geometries are proposed for different kind of situations. The path losses in the reference geometries of the ECC report 159 are calculated as free space losses with certain assumptions of the used antennas. This paper describes two measurement campaigns: one studying the outdoor portable WSD to fixed rooftop DTT reference geometry and one studying indoor portable to indoor portable reference geometry.

\section{A. Reference geometries}

The outdoor reference geometry considered in this work is for rooftop antenna DTT reception at $10 \mathrm{~m}$ height with a portable WSD at $1.5 \mathrm{~m}$ height $22 \mathrm{~m}$ away from the DTT receiver site [4]. An omnidirectional antenna is assumed for the WSD so that a polarization discrimination of $3 \mathrm{~dB}$ is possible, but in the measurements a directional antenna with horizontal polarization for the WSD was also used to increase the available WSD equivalent isotropically radiated power (EIRP). The DTT-antenna specified in the geometry has a gain of $+9.15 \mathrm{dBi}$ (the antenna gain with respect to the hypothetical isotropic antenna) which is assumed to drop by $0.45 \mathrm{~dB}$ to the direction of the WSD $22 \mathrm{~m}$ away. The antenna is described in the ITU-R BT 1368 [8]. This geometry aims to represent a worst-case scenario: if the WSD is situated closer, the antenna gain will drop, and if the WSD is further away the free space loss will increase, decreasing the interfering signal power at the DTT receiver. The considered outdoor reference geometry is illustrated in Figure 1.

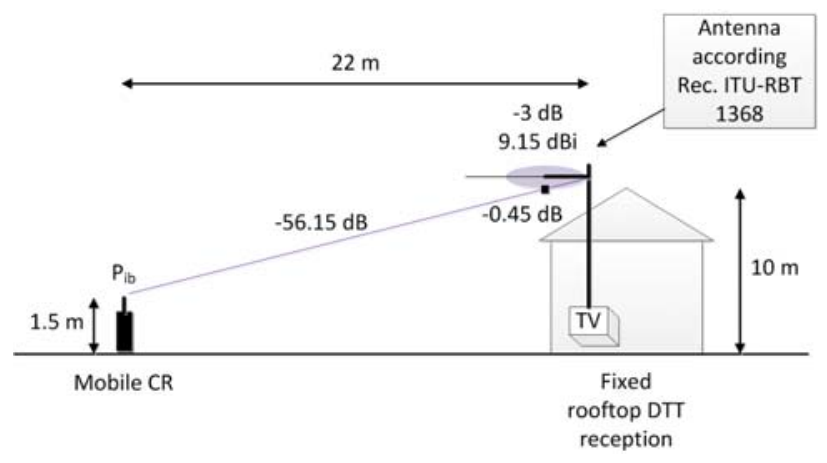

Fig. 1. Outdoor reference geometry

The portable indoor reference geometry considered in this work is shown in Figure 2. Both the DTT reception antenna and the WSD transmitter are at $1.5 \mathrm{~m}$ height $2 \mathrm{~m}$ away from each other. Omnidirectional antennas are assumed for both the WSD and DTT with no polarization discrimination. This was repeated in the measurements by using two similar omnidirectional antennas with a nominal gain of $2 \mathrm{dBi}$.

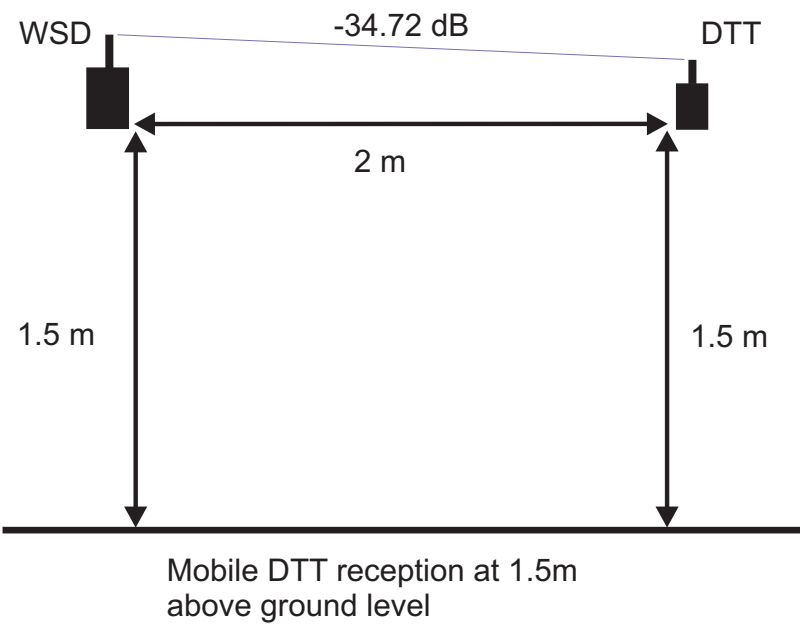

Fig. 2. Indoor reference geometry

\section{B. Outline and contributions of the paper}

During the last few years, several measurement campaigns regarding the use of white space devices in TV frequencies have been conducted all over the world. To give a few examples, they have investigated white space availability through spectrum occupancy measurements and experimental verification of prediction models [9] [10], detection thresholds and sensing performance of white space devices [11] [12], and test beds for white space device networks [13]. However, these efforts have not been able to clearly give safe operational rules for white space devices. The approach in CEPT ECC SE43 for laying a technical basis for white space devices consists of the above-mentioned reference geometries. In this paper we evaluate practical limits for WSD operation in such geometries.

In the following we first in Section II describe the DTT test network, signal configurations, and measurement equipment used in the work. In Section III we outline the method used for measuring co- and adjacent channel protection ratios in the outdoor geometry with varying DTT signal strength, and summarize the obtained numerical results. In Section IV we describe the indoor geometry locations and measurements, discuss interference propagation characteristics observed in the measurements, and list the protection ratios observed in the indoor cases. We summarize and conclude the paper in Section V.

The primary objective of this paper is to provide realistic numerical estimates on the maximum transmitted power of a white space device possible without visible errors in a digital television receiver situated according to the reference geometries. This is done for practical DVB-T and DVBT2 signal configurations, various DTT receiver locations and signal strengths within the test network, and several cases 
TABLE I

TEST NETWORK TRANSMITTERS

\begin{tabular}{|c|c|c|}
\hline & Transmitter 1 & Transmitter 2 \\
\hline Measurement & Outdoor & Indoor \\
\hline Frequency & $610 \mathrm{MHz}$ & $610 \mathrm{MHz}$ \\
\hline Effective radiated power & $250 \mathrm{~W}$ & $1 \mathrm{~kW}$ \\
\hline Antenna height from GL & $92 \mathrm{~m}$ & $40 \mathrm{~m}$ \\
\hline Polarisation & Horizontal & Horizontal \\
\hline
\end{tabular}

of frequency separation between the WSD and DTT signals. Furthermore, the given results can be extended to estimate protection ratios for other primary and secondary transmission systems or signal types if their spectral characteristics are known. We consider these results to be useful for regulatory work regarding the application of white space devices over broadcast networks, and also for cognitive radio algorithm and system development.

\section{Measurement Setup}

\section{A. Test network and DTT signal configurations}

The measurement campaigns of this paper were conducted in a DTT test network operating in Turku, Finland. The network consists of three transmitters, two of which are operable as a single frequency network (SFN). The network covers a relatively large geographical area and covers diverse environments including urban, suburban and rural locations.In the measurements presented in this paper, two of the transmitters were used separately. Specifications of the transmitters used in the measurements are given in Table I. Signal strength maps of the indoor and outdoor measurements are shown with the transmitter locations and sites used for the reference geometry measurements marked in Figures 3 and 4. External view of the building used in the indoor measurements is shown in Figure 5.

In the outdoor measurements DTT transmitter 1 was used to transmit a DVB-T signal [14] with the following parameters:

- $\mathrm{FFT}=8 \mathrm{k}$

- Guard Interval = $1 / 8$

- Modulation $=64-\mathrm{QAM}$, Code rate $(\mathrm{CR})=2 / 3$

DTT Transmitter 2 located in the city centre was used in the indoor measurements to transmit a DVB-T2 signal [15] with the following parameters:

- $\mathrm{FFT}=32 \mathrm{k}$, extended (i.e. the guard band is reduced by making some of the guard carriers active)

- Guard Interval $=1 / 32$, Pilot pattern $=4$

- Modulation $=256-\mathrm{QAM}$, Code rate $=3 / 5$

- Rotated constellation

\section{B. Measurement Equipment}

1) DTT receivers: Two different DTT receivers were used in the measurements. Most of the measurements were performed using a modern integrated TV set with DVB-T2 capability. In the outdoor measurements a set top box was

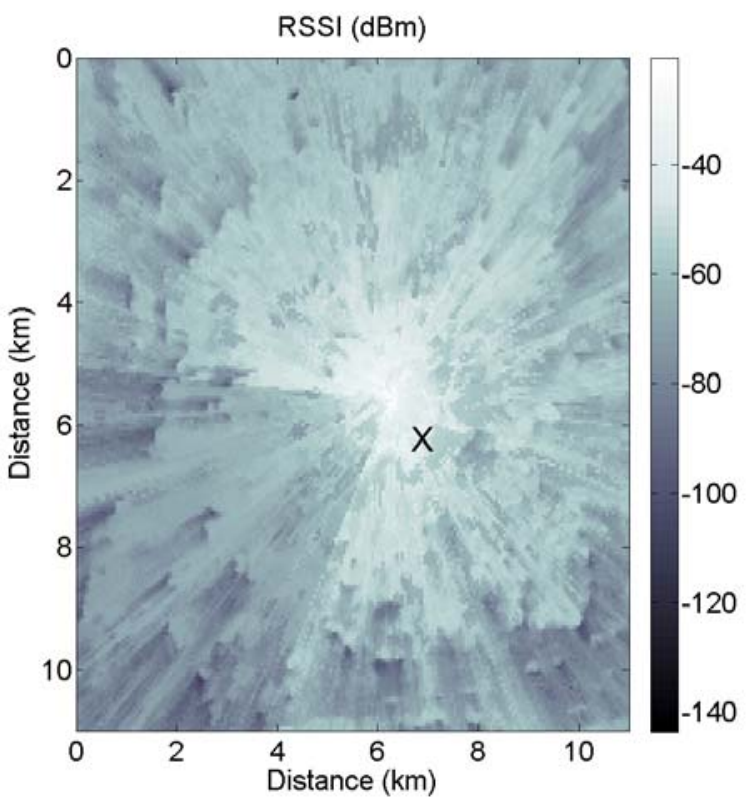

Fig. 3. Indoor measurement location

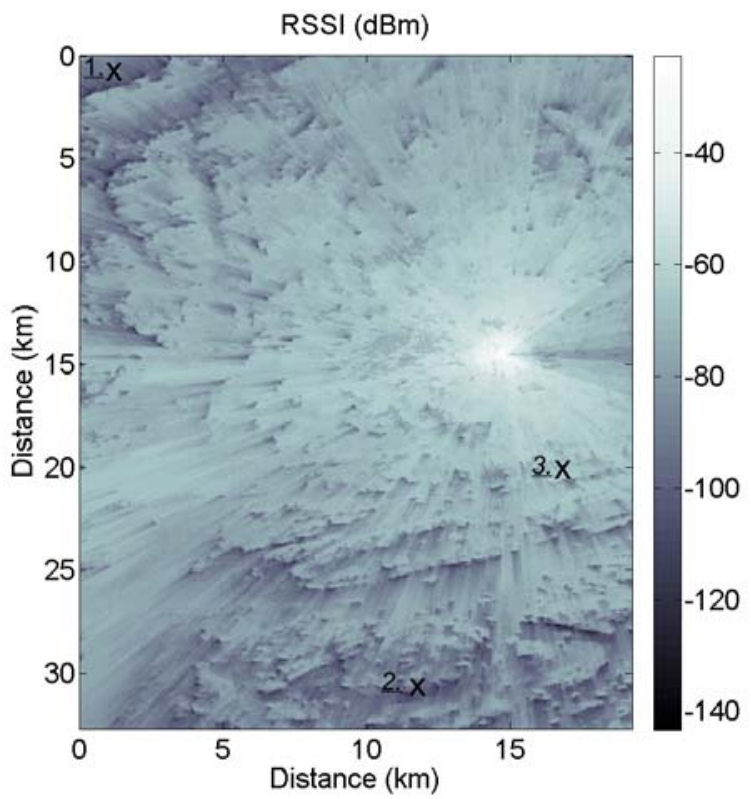

Fig. 4. Outdoor measurement locations: 1) $-80 \mathrm{dBm}$ level 2) $-70 \mathrm{dBm}$ level 3) $-62 \mathrm{dBm}$ level

used for comparison. The integrated set has a sensitivity of -83 $\mathrm{dBm}$ for DVB-T 64QAM 2/3 signals over a Gaussian channel and a sensitivity of $-83.3 \mathrm{dBm}$ for the DVB-T2 32k 256QAM 3/5 signal over a Gaussian channel. These sensitivity levels were used as a reference for the measurement setup. In practice the reception level was selected so that the TV set was operating $3 \mathrm{~dB}$ above the sensitivity. The characteristics of the TV sets were also measured in the laboratory for different channel conditions. 
TABLE II

OUTDOOR PROTECTION RATIOS WITH 64-QAM DVB-T

\begin{tabular}{|c|c|c|c|c|c|c|c|c|c|}
\hline \multirow{2}{*}{ Frequency (MHz) / (Channel) $)$} & \multicolumn{3}{|c|}{ DVB-T level -80 $\mathrm{dBm}$} & \multicolumn{2}{c|}{ DVB-T level -70 dBm } & \multicolumn{3}{c|}{ DVB-T level -62 $\mathrm{dBm}$} \\
\cline { 2 - 12 } & WSD & PR & WSD & \multicolumn{2}{l|}{ PR } & WSD & PR \\
\cline { 2 - 11 } & $\begin{array}{c}\text { EIRP } \\
(\mathrm{dBm})\end{array}$ & $\begin{array}{c}\text { FS } \\
(\mathrm{db} \mu \mathrm{V} / \mathrm{m})\end{array}$ & $\begin{array}{c}\text { EIRP } \\
(\mathrm{dB})\end{array}$ & $\begin{array}{c}\text { FS } \\
(\mathrm{dBm})\end{array}$ & $\begin{array}{c}\text { EIRP } \\
(\mathrm{db} \mu \mathrm{V} / \mathrm{m})\end{array}$ & $\begin{array}{c}\text { FS } \\
(\mathrm{dB})\end{array}$ & $\begin{array}{c}\mathrm{dBm}) \\
(\mathrm{db} \mu \mathrm{V} / \mathrm{m})\end{array}$ & $(\mathrm{dB})$ \\
\hline $578 /(\mathrm{N}-4)$ & 18.9 & 94.0 & 48.4 & 32.5 & 107.6 & 51.9 & 36.4 & 111.5 & 49.0 \\
\hline $586 /(\mathrm{N}-3)$ & 18.3 & 93.4 & 47.5 & 21.5 & 96.6 & 40.6 & 23.0 & 98.1 & 35.4 \\
\hline $594 /(\mathrm{N}-2)$ & 15.2 & 90.2 & 44.1 & 18.7 & 93.7 & 38.4 & 20.5 & 95.5 & 33.2 \\
\hline $602 /(\mathrm{N}-1)$ & 1.7 & 77.0 & 31.4 & 13.3 & 88.6 & 33.2 & 12.8 & 88.1 & 25.9 \\
\hline $610 /(\mathrm{N})$ & -50.0 & 24.7 & -19.7 & -36.5 & 38.2 & -16.2 & -30.0 & 44.7 & -17.7 \\
\hline $682 /(\mathrm{N}+9)$ & 20.9 & 95.6 & 51.1 & 33.8 & 108.5 & 53.7 & 38.5 & 113.2 & $\mathbf{4 8 . 4}$ \\
\hline
\end{tabular}

2) WSD Tx signal generation: The WSD signal was simulated with a constant OFDM signal from a DVB-T signal generator. This setup was chosen because the primary focus of this work is on the WSD uplink, and using a constant signal, power level measurements could be done very reliably with a TV signal analyzer. The signal bandwidth was $7.6 \mathrm{MHz}$, filling the entire DTT broadcast channel. The protection ratio of the WSD signal towards the DTT receivers was measured in all configurations. Using the co-channel protection ratio, the Adjacent Channel Leakage Ratio (ACLR) of the WSD signal and the Adjacent Channel Selectivity (ACS) of the DTT receiver these results can be scaled to any other signal as shown in Annex 3 of [4].

The used signal generator was able to provide output power levels up to $+20 \mathrm{dBm}$, which was considered to be adequate in the indoor conditions and no extra power amplifier was used. More power was needed in the outdoor measurements and an additional power amplifier was added to the setup. The power amplifier has a gain of $40 \mathrm{~dB}$ and maximum output power of approximately $10 \mathrm{~W}$. As the thermal noise level of the power amplifier is rather high, step attenuators were used at the output of the power amplifier so that full control of the WSD signal including the sideband noise was possible. This was important as the target was to maintain a constant ACLR for the signal during the power level setting.

3) Receiving and transmitting antennas in the indoor measurements: In the basic measurement setup two similar professional $2 \mathrm{dBi}$ omnidirectional antennas were used. These were set to $1.5 \mathrm{~m}$ height. The antennas are specified for the entire UHF broadcasting band, but the radiation patterns vary slightly over the band. In indoor conditions with relatively small rooms it is very difficult to distinguish between the antenna gain to a certain direction at a given frequency and the effects of the radio channel. Therefore in the measurements a direct coupling between the antennas was measured instead of the path loss. In the measurements the antennas were kept in fixed angular position towards each other, so that the same sides were always facing each other.

4) Receiving and transmitting antennas in the outdoor measurements: For the receiving antenna a typical consumergrade Yagi antenna was used. The nominal announced gain for this antenna is $12.5 \mathrm{dBi}$, but the antenna is very wide band and the gain variations within the band are not known. For path loss measurements the antenna gain towards the WSD transmitter (20 degrees downwards) was calibrated with a professional antenna with a gain of $12 \mathrm{dBi}$ and a specified frequency range of 585 - $635 \mathrm{MHz}$. A similar comparison was done with the DVB-T signal and the professional antenna in the main beam. This would indicate that the gain of the consumer-grade Yagi is around $9.3 \mathrm{dBi}$ at $610 \mathrm{MHz}$. The professional $12 \mathrm{dBi}$ Yagi antenna was also used to increase the directional transmitted power of the WSD transmitter.

5) Receivers: All the power and spectrum measurements were performed using a TV signal analyzer which enables measuring accurately the channel power of a DVB-T2 signal as well as performing normal spectrum measurements. In the outdoor measurements two analyzer devices were available, which made it possible to monitor the power levels in both $\mathrm{Tx}$ and Rx sides. Power levels were adjusted in $1 \mathrm{~dB}$ steps in all measurements and received power levels were recorded with one decimal accuracy.

\section{Outdoor Field MEAsurements}

The outdoor measurements studied the maximum possible WSD power in the corresponding reference geometry before the received picture failed. Thus, the measured protection ratio between DTT reception at center frequency $f_{p r i}$ and transmitted WSD signal at center frequency $f_{w s d}$ becomes

$$
P R\left(f_{p r i}, f_{w s d}\right)=\frac{P_{p r i}\left(f_{p r i}\right)}{P_{w s d, \max }\left(f_{w s d}\right)}
$$

The measurements were done at three different field strengths. The first one was at an input level of $-80 \mathrm{dBm}$, $3 \mathrm{~dB}$ from the sensitivity limit of the receiver, corresponding to the situation where the DTT receiver is located at the edge of the service area. The second one was with an input level of $-70 \mathrm{dBm}$, at $10 \mathrm{~dB}$ higher level than the first one, and the last one with $-62 \mathrm{dBm}$ input level, $18 \mathrm{~dB}$ higher than the first one; the highest signal level was limited by the maximum WSD transmission power it was possible to use in the measurements. The locations of the measurements were selected so that a suitable signal level was achieved with the Yagi antenna at 
TABLE III

INDOOR PROTECTION RATIOS WITH 256-QAM DVB-T2

\begin{tabular}{|c|c|c|c|c|c|}
\hline \multirow{2}{*}{ Frequency (MHz) / (Channel) } & \multicolumn{5}{|c|}{ Protection ratio with 32k 256-QAM 3/5 in room } \\
\cline { 2 - 6 } & Muo 2m & L229 2m & C20 2m & L321 2m & Average \\
\hline $578 /(\mathrm{N}-4)$ & 43.0 & $\mathbf{4 0 . 1}$ & 43.9 & 44.5 & 42.9 \\
\hline $586 /(\mathrm{N}-3)$ & 41.9 & 42.5 & 42.9 & 40.1 & 41.9 \\
\hline $594 /(\mathrm{N}-2)$ & 39.5 & 40.8 & 40.9 & 38.9 & 40.0 \\
\hline $602 /(\mathrm{N}-1)$ & 25.6 & 28.6 & 35.0 & 30.7 & 30.0 \\
\hline $610 /(\mathrm{N})$ & -23.5 & -23.6 & -22.8 & -23.8 & -23.4 \\
\hline
\end{tabular}

the $10 \mathrm{~m}$ height. The measurement locations in relation to the test network DTT transmitter are illustrated in Figure 4.

Interference measurements were performed with the WSD operating on the co-channel $(N)$ and adjacent channels $N-1$, $N-2, N-3 N-4$ and $N+9$. The WSD transmission power was increased until errors were detected in the picture and then decreased by $1 \mathrm{~dB}$ steps until no errors were visible during an observation period of several tens of seconds, corresponding roughly to the ESR5 criterion [2]. Then the power fed to the WSD transmission antenna was measured in the co-channel case. In the adjacent channel cases, the received WSD interference power at the DTT receiver input was also measured and used to calculate the corresponding protection ratio. It should be noted that with the WSD operating on the adjacent channel $N+9$ in the scenario with DTT signal strength -62 $\mathrm{dBm}$, the WSD transmission power was insufficient to produce errors in the DTT reception, and the true protection ratio is higher than given here. This case is marked with bold text in the Table.

A summary of the maximum WSD power levels for the different frequencies and DTT input levels are shown in Table II. Also the corresponding Field Strength (FS) at the DVB-T reception site is shown as well as the calculated protection ratio from (2). The ACLR values for each measurement and the laboratory measurements of the DTT-receivers can be found in [16].

\section{INDOOR FIELD MEASUREMENTS}

\section{A. Location}

All indoor measurements were performed in an old school building constructed mainly of brick and concrete, whose windows are without any metallised shielding. It should be noted that DTT transmissions penetrate this type of building better than a modern office building. The following rooms were used for the measurements:

- "Muotoilutila" (Muo): a small rooftop space used for project works.

- L229: class room in L-wing, second floor

- C20: class room in C-wing, second floor

- L321: class room in L-wing, third floor

The "Muotoilutila" is a small room on top of the C-wing and has a window towards the transmitter. All other rooms have windows perpendicular to the transmitter direction, so no direct signal was received in these cases. The building, measurement locations, and transmitter direction are illustrated in Figure 5.

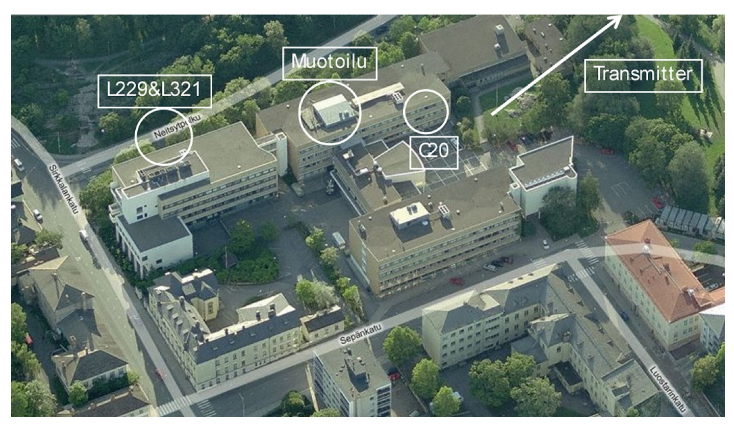

Fig. 5. Indoor measurement premises

\section{B. Coupling losses}

The rooms where the measurements were performed have a different geometry and thus different reflections from the surroundings. Therefore the coupling losses also differ between the rooms even though the distance between the WSD and DTT is constant. The coupling losses were measured in each room in the $2 \mathrm{~m}$ reference geometry. A comparison between these and free-space loss is shown in Figure 6. Even though the number of measurements is not large enough to give statistically reliable results, a clear trend in the measurements is that the coupling loss is likely to be higher than the free-space loss (FSL). Worst-case scenarios where the reflections actually would significantly reduce the coupling loss between the WSD and DTT, that is, amplify the interference from the WSD to the DTT, seem unlikely in light of these measurements, although not enough coupling loss measurements were performed to produce a comprehensive study on the matter.

\section{WSD maximum power and protection ratios}

In the indoor measurements we again studied the maximum possible WSD power in the reference geometry before the DTT reception failed. Most of the measurements were done close to the sensitivity limit corresponding to the situation where the DTT receiver is located at the edge of the service area or in otherwise difficult indoor conditions. The receiver sensitivity level was found by increasing the attenuation of the received TV signal using a step attenuator until picture 


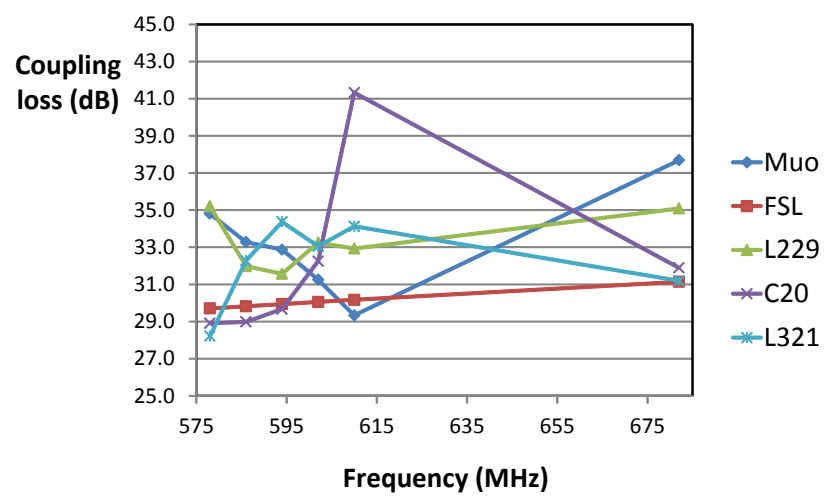

Fig. 6. Free-space loss and coupling losses in different rooms

failure. After that the signal level was increased by $1 \mathrm{~dB}$, so that no errors were observed in the picture and this was taken as the sensitivity level in the channel conditions of the receiver location. From this level the wanted measurement level was obtained by increasing the signal level by $3 \mathrm{~dB}$. Again the transmission power of the WSD was increased until errors were detected in the picture and then decreased by $1 \mathrm{~dB}$ step so that no errors were visible during an observation period of several tens of seconds.

The protection ratios were calculated from the measured WSD and DTT powers at the TV set input. Results are shown in Table III. In one measurement (L $2292 \mathrm{~m} / \mathrm{N}-4$ ) the available WSD power was not enough to cause any errors, thus the calculated protection ratio is a minimum and the real value may be higher. This case is marked with bold text in the Table.

The protection ratio values are rather consistent if the unreliable points are excluded. The greatest spread is in the values at $N-1(602 \mathrm{MHz})$. On average the co-channel value is between -20 and $-25 \mathrm{~dB}$, on channel $N-1$ between 25 and 35 $\mathrm{dB}$, on $N-2$ between 39 and $42 \mathrm{~dB}$, and on $N-3$ and $N-4$ between 40 and $45 \mathrm{~dB}$. The variations are at least partly due to differing channel conditions in the different locations. Average protection ratios for the indoor measurements are shown in Table III. The laboratory measurements performed to calibrate the measurement setup are explained in [17].

\section{CONCLUSION}

In this paper we have presented results of a measurement campaign conducted in a digital terrestrial television test network with DVB-T and DVB-T2 signal configurations. White space device interference was measured over previously proposed outdoor and indoor reference geometry scenarios; based on these measurements, realistic numerical estimates have been provided on the maximum transmitted power of a white space device possible without visible errors in a digital television receiver situated according to the reference geometries. This has been done for various DTT receiver locations and signal strengths within the test network, and several cases of frequency separation between the WSD and DTT signals. The obtained protection ratios are also scalable to other signals with known characteristics. The presented work can be considered as the first practical testing of the proposed theoretical reference WSD scenarios, but also as a source of realistic numerical estimates for the minimum protection ratios between WSD transmitters and primary TV receivers; as such, the given data can be used for example in further WSD system development, simulation, and performance evaluation.

\section{ACKNOWLEDGEMENTS}

This work was funded by Tekes in the Trial program, and by the academy of Finland (133888).

\section{REFERENCES}

[1] ECC report 148, "Measurements on the performance of DVB-T receivers in the presence of interference from the mobile service", June 2010. Available at urlhttp://www.erodocdb.dk/

[2] ITU-R Document 6E/64-E, "The ESR5 criterion for the Assessment of DVB-T transmission Quality", April 2004.

[3] CEPT/ECC SE43 - Cognitive Radio Systems - White Spaces. http:// www.cept.org/ecc/groups/ecc/wg-se/se-43/.

[4] ECC report 159, "Technical and operational requirements for the possible operation of cognitive radio systems in the 'white spaces' of the frequency band 470-790 MHz", January 2011. Available at www.erodocdb.dk/

[5] Federal Communication Commision, "Second Report and Order and Memorandum Opinion and Order In the Matter of Unlicensed Operation in the TV Broadcast Bands, Additional Spectrum for Unlicensed Devices Below $900 \mathrm{MHz}$ and in the $3 \mathrm{GHz}$ Band", Document 08-260, November 2008.

[6] Ofcom, "Implementing Geolocation - Summary of consultation responses and next steps", September 2011. Available at http:// stakeholders.ofcom.org.uk/consultations/geolocation/

[7] J. Ojaniemi, J. Poikonen, R. Wichman, "Effect of geolocation database update algorithms to the use of TV white spaces", Submitted for review to the 7 th International Conference on Cognitive Radio Oriented Wireless Networks \& Communications (CROWNCOM), Stockholm, June 2012.

[8] ITU-R BT.1368-8, "Planning criteria for digital terrestrial television services in the VHF/UHF bands", 2008.

[9] E. Obregon, L. Shi, J. Ferrer and Jens Zander, "Experimental Verification of Indoor TV White Space Opportunity Prediction Model", Proc. The Fifth International Conference on Cognitive Radio Oriented Wireless Networks \& Communications (CROWNCOM), Cannes, France, June 2010.

[10] H. Islam et. al, "Spectrum Survey in Singapore: Occupancy Measurements and Analyses", Proc. The Third International Conference on Cognitive Radio Oriented Wireless Networks \& Communications (CROWNCOM), Singapore, May 2008.

[11] M.A. McHenry, K. Steadman and M. Lofquist, "Determination of Detection Thresholds to Allow Safe Operation of Television Band "White Space" Devices", Proc. The 3rd IEEE Symposium on New Frontiers in Dynamic Spectrum Access Networks (DySPAN), Chicago, The USA, October 2008.

[12] Ser Wa Oh et. al, "TV White-Space Video Streaming Demo", Proc. IEEE Symposium on New Frontiers in Dynamic Spectrum Access Networks (DySPAN) 2010, Singapore, April 2010.

[13] X. Feng, J. Zhang and Q. Zhang, ”Database-Assisted Multi-AP Network on TV White Spaces: Architecture, Spectrum Allocation and AP Discovery", Proc. IEEE Symposium on New Frontiers in Dynamic Spectrum Access Networks (DySPAN) 2011, Aachen, Germany, May 2011.

[14] ETSI EN 300744 V1.6.1., "Digital Video Broadcasting (DVB); Framing structure, channel coding and modulation for digital terrestrial television", January 2009.

[15] ETSI EN 302755 V1.1.1, "Frame structure channel coding and modulation for a second generation digital terrestrial television broadcasting system (DVB-T2)", September 2009.

[16] "CEPT/ECC SE 43(11)36 Wise-Project Measurement Report: WSD maximum Power Measurements in Turku Test Network",10th SE43 meeting, Bologna, Italy, July 2011. Available at http://cept.org

[17] "CEPT/ECC SE43(11)81 Wise-Project Measurement Report: WSD maximum Power Indoor Measurements in Turku Test Network", 12th SE43 meeting, Cambridge, UK, December 2011. Available at http: //cept.org 